IdeAs

Idées d'Amériques

$18 \mid 2021$

Frontières dans les Amériques - Intégration, sécurité et migrations

\title{
Michael Welsh, Big Bend National Park: Mexico, the United States and a Borderland Ecosystem
}

Reno, University of Nevada Press, 2021

Jean-Daniel Collomb

\section{OpenEdition \\ Journals}

Édition électronique

URL : https://journals.openedition.org/ideas/11873

DOI : $10.4000 /$ ideas. 11873

ISSN : 1950-5701

Éditeur

Institut des Amériques

Référence électronique

Jean-Daniel Collomb, « Michael Welsh, Big Bend National Park: Mexico, the United States and a

Borderland Ecosystem », IdeAs [En ligne], 18 | 2021, mis en ligne le 01 octobre 2021, consulté le 21

octobre 2021. URL : http://journals.openedition.org/ideas/11873; DOl : https://doi.org/10.4000/ideas. 11873

Ce document a été généré automatiquement le 21 octobre 2021

\section{(c)}

IdeAs - Idées d'Amériques est mis à disposition selon les termes de la licence Creative Commons Attribution - Pas d'Utilisation Commerciale - Pas de Modification 4.0 International. 


\section{Michael Welsh, Big Bend National Park: Mexico, the United States and a Borderland Ecosystem}

Reno, University of Nevada Press, 2021

Jean-Daniel Collomb

\section{RÉFÉRENCE}

Michael Welsh, Big Bend National Park: Mexico, the United States and a Borderland Ecosystem, Reno, University of Nevada Press, 2021, 214 p.

1 Michael Welsh consacre un ouvrage au parc national de Big Bend, situé dans l'État du Texas, à la frontière avec le Mexique. Cette étude, qui s'inscrit dans le champ de l'histoire environnementale (à la confluence des dynamiques sociales et culturelles, et des influences physiques et biologiques) retrace l'histoire de cette région et s'intéresse aux origines du parc et à l'évolution de sa gestion. Le livre contient sept chapitres agrémentés de cartes et d'illustrations utiles pour le lecteur.

2 Une courte introduction présente les caractéristiques géographiques et environnementales de la région. Dès le début, on retrouve la polyvalence et le caractère pluridisciplinaire de l'histoire environnementale à travers une problématisation incluant la géopolitique et les identités culturelles.

Dans le premier chapitre, l'auteur insiste sur les spécificités physiques de Big Bend qui façonnent son histoire et imposent des contraintes à l'action humaine en son sein. La zone se caractérise par son aridité et son isolement, puisqu'elle est composée à $98 \%$ du désert de Chihuahua. Les Espagnols peinent à contrôler une région qu'ils connaissent mal et où les Comanches imposent leur loi aux XVII ${ }^{e}$ et XVIII ${ }^{e}$ siècles. Ce chapitre nous rappelle à quel point il a été difficile pour les États, avant le développement des bureaucraties modernes, de connaître et de maîtriser les espaces qui composent leurs 
territoires. Welsh montre bien que ce constat vaut aussi bien sous l'empire espagnol que sous l'autorité du Mexique, puis des États-Unis.

4 Le chapitre suivant place l'intérêt grandissant pour l'idée d'un parc national de Big Bend dans le contexte des premières décennies du National Park Service américain (fondé en 1916). Il souligne le rôle du patriotisme, l'intérêt pour la science et l'archéologie, ainsi que l'influence des vulgarisateurs qui s'efforcent d'attirer l'attention des décideurs et du public vers cette région difficilement accessible. Le trait distinctif de Big Bend réside dans l'éventualité (encore hypothétique à ce jour) d'un parc binational conjointement administré par les États-Unis et le Mexique. Le président Franklin Roosevelt y est favorable et des hauts fonctionnaires des deux pays se mobilisent pour faire avancer cette idée.

Dans le troisième chapitre, Welsh entre davantage dans les détails des négociations menées dans les années 1930. Il montre la valeur archéologique de Big Bend et l'intérêt de la Wildlife Division du National Park Service pour la faune et la flore de la région. Dans un second temps, il passe en revue les questions qui occupent les représentants des deux pays : quid de la coupe du bois par des citoyens mexicains? De la réglementation de la pêche dans le Rio Grande ? Du bétail qui traverse le fleuve depuis le Mexique?

Dans le chapitre suivant, il est à nouveau question de l'idée d'un parc international. L'un des mérites de ce chapitre est de s'appesantir sur le rôle joué par le Mexique, notamment à travers l'engagement du président Lázaro Cárdenas dans les années 1930. Le Mexique crée ses premières zones protégées pour des raisons semblables à celles qui avaient conduit les États-Unis à établir des parcs et des forêts nationales dès le xix siècle. Welsh fait brièvement allusion à l'influence du conversationniste Gifford Pinchot sur ses homologues mexicains, mais on regrettera qu'il n'ait pas évoqué les retentissements éventuels sur le Mexique des tensions entre conservationnistes utilitaires (menés par Pinchot) et partisans des parcs nationaux (auxquels Pinchot était hostile). Ici, la recherche d'une gestion éclairée des ressources, le tourisme et l'intérêt pour l'histoire sont présentés comme formant un tout non-problématique.

7 Alors que la création d'un parc international semblait en bonne voie, la situation se dégrade rapidement à partir de 1937, au moment où le gouvernement mexicain décide de se consacrer à des politiques redistributives volontaristes et où l'État fédéral américain se préoccupe de plus en plus du contexte international. Si le parc national de Big Bend voit bien officiellement le jour en 1944 du côté états-unien de la frontière, la création du parc international n'est plus d'actualité.

8 Dans le cinquième chapitre, Welsh décrit la gestion du parc dans les décennies qui suivent le second conflit mondial. Il évoque notamment la volonté des administrateurs de favoriser une expérience "authentique" du temps des pionniers, ainsi que l'augmentation de la popularité du parc à la suite d'une visite médiatisée de Lady Bird Johnson en 1966. L'auteur note également la tension entre la popularité nécessaire à la pérennisation du parc et les risques environnementaux que comporte une éventuelle sur-fréquentation liée au tourisme de masse.

9 Le chapitre suivant se concentre sur la part sombre de la gestion du parc durant la seconde partie $d u x^{e}$ siècle. L'auteur présente les défis redoutables auxquels les gestionnaires du parc sont confrontés : immigration et commerce illégaux, traversées illégales de la frontière par le bétail venu du Mexique et surtout trafic de drogue. Les autorités américaines développent une approche de plus en plus répressive, qui relègue 
l'idée d'un parc international (encore vivace dans certains esprits) au rang de chimère. Selon Welsh, Big Bend redevient alors partiellement la zone de tensions qu'il avait été à la fin du XIXe siècle avant le rapprochement entre les États-Unis et le Mexique.

Dans l'ultime chapitre, Welsh relève que les relations entre les gouvernements des deux pays s'améliorent à partir des années 1980, au point de redonner espoir aux partisans du parc international. Mais une fois encore, la réalité géopolitique porte un coup d'arrêt à une dynamique qui commençait à ressembler à celle du milieu des années 1930. Les attentats du 11 septembre 2001 conduisent l'administration Bush à durcir le contrôle de sa frontière méridionale. L'idée d'un parc international ne meurt pas, mais se heurte désormais à l'hostilité de nombreux élus républicains qui se disent soucieux de la sécurité nationale des États-Unis.

11 Cet ouvrage est le produit d'un travail de recherche très méticuleux, qui s'appuie sur l'étude approfondie de nombreuses archives. Il sera donc très utile aux lecteurs désireux de connaître précisément l'histoire de ce parc. En revanche, on pourra déplorer que l'auteur privilégie le plus souvent la narration aux dépens de l'analyse. À la lecture de cet ouvrage, il semble par exemple que l'idée de créer un parc ait découlé de la volonté d'un nombre restreint d'individus et que, parmi eux, les élus et les hauts fonctionnaires aient joué un rôle central. Il est finalement assez peu question de l'influence de la société civile (même si celle-ci n'est pas absente). On aurait aimé que l'auteur commente cette tendance et son éventuelle originalité en comparaison d'autres parcs.

\section{AUTEURS}

\section{JEAN-DANIEL COLLOMB}

Jean-Daniel Collomb est professeur des universités en civilisation américaine à l'Université Grenoble-Alpes. Ses recherches portent sur les mouvements sociaux, guerre et société, l'intersection de l'art (en particulier la musique populaire) et de la politique, et les jeunes souscultures.Jean-Daniel.Collomb@univ-grenoble-alpes.fr 